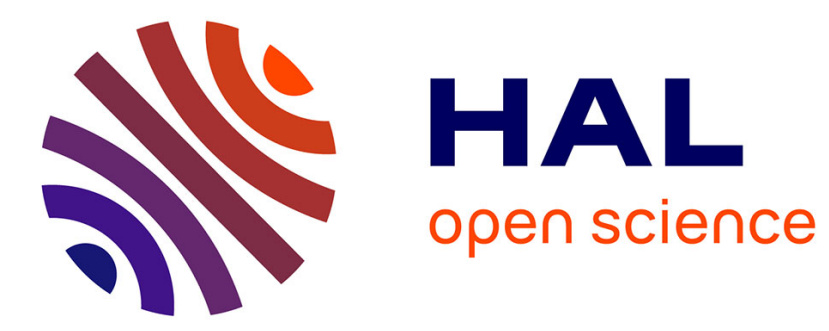

\title{
Le réexamen des lois de bioéthique, pour quel monde commun?
}

Jean-Philippe Pierron

\section{To cite this version:}

Jean-Philippe Pierron. Le réexamen des lois de bioéthique, pour quel monde commun?. Revue Esprit, 2021. hal-03271988

\section{HAL Id: hal-03271988 \\ https://hal.science/hal-03271988}

Submitted on 28 Jun 2021

HAL is a multi-disciplinary open access archive for the deposit and dissemination of scientific research documents, whether they are published or not. The documents may come from teaching and research institutions in France or abroad, or from public or private research centers.
L'archive ouverte pluridisciplinaire HAL, est destinée au dépôt et à la diffusion de documents scientifiques de niveau recherche, publiés ou non, émanant des établissements d'enseignement et de recherche français ou étrangers, des laboratoires publics ou privés. 


\section{Le réexamen des lois de bioéthique, pour quel monde commun?}

\section{Jean-Philippe Pierron}

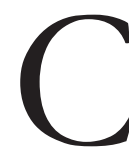

omment une société fait-elle le point sur ce qui, pour elle, compte au point qu'elle ne saurait, en ces matières, transiger? Peut-elle autre, dans le flux et la vitesse, des stases pour se livrer à un auto-examen? N'est-ce d'ailleurs pas cela l'éthique, dans la bioéthique: offrir collectivement le scrupule de la pause au cœur de la trépidation des courses à l'innovation? Elle peut le faire en imagination, de façon prospective, en se projetant dans le temps. La récente réinitialisation d'un HautCommissariat au Plan servira une telle entreprise. Elle peut le faire à travers un travail de mémoire, consistant à revisiter les actions menées, les évolutions survenues, les questions de société qui sont progressivement apparues et qui méritent d'être débattues, en vue de la suite à leur donner. Le réexamen obligatoire depuis la loi de 2011, une fois tous les sept ans, de la loi relative à la bioéthique, précédé d'un débat public sous forme d'états généraux, travaille à cette intention, cherchant à répondre à la question: «Quel monde voulons-nous pour demain?» 


\section{Superbe et douloureuse réforme des lois bioéthiques}

On ne peut négliger, on doit même saluer la réflexivité par le biais de laquelle la société française s'est mise en travail. Se réunir en états généraux tout d'abord avant de le faire en assemblée, pour le temps d'une soirée ou d'un après-midi; penser et réfléchir ensemble, experts et non-experts sur des enjeux aussi sensibles et complexes que l'assistance médicale à la procréation (AMP), les liens entre l'intelligence artificielle et le soin, les relations entre santé et environnement, les modes de gestion de la santé, comme cela a pu être le cas dans toute la France de 2018 à 2021 n'est ni anodin, ni dérisoire.

Il y a là une forme de grandeur que trop vite on écrasera, n'y voyant qu'une forme de manipulation des opinions, un mirage de démocratie participative à travers des consultations en ligne. Une communauté historique se donne là l'occasion de préciser et de se redire à elle-même et à l'ensemble de ses concitoyens, dans la conscience de ce qui la solidarise et la distingue des autres sociétés et États, quelles sont ses obstinations durables et ce qu'elle cherche à faire vivre. Mais on doit, dans le même temps, formuler quelques étonnements. La première observation est de vocabulaire. L'expression même de « loi de bioéthique » est équivoque: s'agit d'éthique ou s'agit-il de droit? Mobilise-t-on des questions qui intéressent la réflexion éthique sur le «quoi faire pour bien faire dans une recherche de la vie bonne » ou des interrogations d'ordre juridique concernant ce qui est légal ou non, licite ou pas? Cette question doit se poser. Outre qu'elle interroge la difficile et délicate frontière entre le déontologique et le juridique, elle questionne davantage encore cette autre frontière sensible qui passe entre éthique et droit. Le risque est de laisser, pour beaucoup, planer le doute sur l'idée que l'éthique ne serait qu'affaire de réglementation - tout ce qui n'est pas illégal est non seulement permis mais légitime -, mettant de côté toute la dimension de discernement éthique engagé en ces matières.

La deuxième observation concerne le principe même de réexamen des lois bioéthiques, mot plus juste que celui de révision. Il laisse planer le doute sur ce que peut être l'affirmation de principes ou d'évaluations fortes quant à ce qui est juste, fraternel ou vrai, si dans le même temps on pose que de telles évaluations seront, non pas caduques, mais objet 
d'un réexamen régulier. Cette non-constance laissant planer le doute sur leur consistance. Du moins interroge-t-elle sur la manière dont nous articulons l'universel des principes et l'historicité de leur traduction.

La troisième perplexité porte sur les relations entre droit et histoire, législation et communauté historique. Si nous avons retenu la prudence d’un Pascal disant: "Vérité en deçà des Pyrénées, erreur au-delà » invitant à ne pas surévaluer les législations nationales, le siècle passé nous a également appris à être prudents à l'égard des philosophies qui affirment brutalement un sens de l'histoire. Convoquer l'existence de lois bioéthiques ratifiées dans d'autres pays comme argument pour légiférer en France est-il le signe que ces derniers seraient en avance alors que celle-ci serait en retard, arguant d'une téléologie de l'histoire dont on ne connait pas l'agenda? Ou bien est-ce là le progrès, que de n'avoir pas avoir légiféré ou refusé de le faire? D'où juge-t-on et selon quel sens de l'histoire pour convoquer qu'une loi serait nécessairement et inéluctablement une loi de progrès? De telles questions sont difficiles lorsqu'il s'agit de se situer à l'intérieur du droit national, alors même que les mobilités, rendues plus aisées aujourd'hui, paraissent s'affranchir des frontières juridiques pour revendiquer la défense des droits personnels.

Enfin, dernière source de perplexité: réexaminer une loi tous les ans est bien ce qui est prévu. Mais pourquoi installer dans cette même vague de réexamen des lois qui, elles, avaient été votées deux ans auparavant (nous pensons aux lois Claeys-Léonetti II 2018 concernant la législation sur la fin de vie, et notamment la question de la sédation profonde et maintenue jusqu'au décès), si ce n'est pour des intérêts dont les motivations mériteraient d'être sondées davantage? Le «sociétal » paraît imposer son rythme, via son retentissement médiatique, eu égard à la temporalité relativement lente du social, qui ne "légifere qu'en tremblant», comme le disait le doyen Carbonnier. Cette question de la temporalité, entre innovation technique, accélération médiatique et réflexion éthique, est loin d'être secondaire dans la manière d'appréhender les enjeux de bioéthique. 


\section{La bioéthique dans des sociétés pluralistes}

Les récentes révisions des lois bioéthiques ont mis à l'agenda de la discussion des enjeux et des thèmes considérables. Rappelons-en les thèmes principaux: cellules souches et recherches sur l'embryon, examens génétiques et médecine génomique, dons et transplantations d'organe, neurosciences, données de santé, intelligence artificielle et robotisation, santé et environnement, procréation et société, prise en charge de la fin de vie. Dans leur diversité apparente, ces thèmes partagent l'idée que la dynamique du vivant peut être maitrisée, au sein du paradigme informationnel: information génétique, information des données massives et intelligence artificielle, gestion de l'information. Outre qu'un tel cadre incite à réduire l'éthique au rang de science de l'action, sous-tendue par des informations « fiables »- décider serait le résultat d'un calcul rationnel -, elles donnent actualité et consistance, s'il en était besoin, à l'affirmation selon laquelle nos sociétés sont complexes, mobiles et différenciées.

Sociétés complexes dans la mesure où, à la croisée de la science, des techniques et de l'industrie, le monde médical, au sens large, initie et crée des situations de vie et d'existence inédites: existence de tiers d'engendrement dans le cadre de l'AMP, levée des secrets de famille avec les tests génétiques et les big data, situations de fin de vie, devenir du soin (care) à l'heure des robots de compagnie, etc. Sociétés mobiles, comme le signale la demande de transcription dans le droit national de pratiques interdites en France (par exemple, la demande de transcription de parentalité à la suite d'une gestation pour autrui) mais légales dans d'autres pays. La situation du pluralisme juridique met en discussion, sinon en compétition, les traditions juridiques nationales à l'heure de la mobilité internationale. Sociétés différenciées, enfin, en raison d'un pluralisme éthique. En matière de bioéthique, une bonne part de la réflexion éthique consiste aujourd'hui à accepter de discuter de ce qui devrait être l'objet de considérations éthiques. Jean-Marc Ferry parle d'une «éthique du débat éthique » dont la valeur tient non pas d'abord aux contenus moraux mais en ce que «la mise en jeu de ces contenus, au cours de débats, suivrait des procédures satisfaisant à un point de vue moral ${ }^{1} \gg$. Or la conscience de ce pluralisme, vécu comme une conquête et non comme un relativisme douteux, peut 
être inquiète. Elle l'est lorsque son dogmatisme et son assurance d'une vision du monde revendiquent des vérités morales indiscutables d'un côté. Elle l'est lorsque l'activisme d'un militantisme des droits personnels exige la reconnaissance de droits non négociables de l'autre. Ces deux tendances ont chacune voulu, pendant les débats bioéthiques, pour des raisons contraires (le bien substantiel au nom du respect de la « nature » versus le bon relatif au nom de l'attention portée aux situations effectives existantes), échapper à cette dimension de l'argumentation et du discutable. Les interventions des régulateurs sur la plateforme de consultation en ligne dans le cadre des États généraux de la bioéthique ont observé la mobilisation de citoyens déjà très concernés (campagnes menées

en faveur de la participation aux États généraux, sur le site d'Alliance $\mathrm{Vita}^{2}$; mobilisation sur les thématiques bioéthiques de communautés très actives en ligne comme l'association $\mathrm{LGBT}^{3}$, La Manif pour tous, l'Association pour le droit de mourir dans la dignité).

\section{Quelques points d'attention}

La réforme des lois bioéthiques contribue-t-elle pour autant à accréditer la thèse selon laquelle nos sociétés seraient, non plus des sociétés traditionnelles assurées de bien faire par le prestige de l'ancestralité, mais des sociétés post-traditionnelles, réflexives, ne cessant de discuter ce qu'elles tiennent pour bien, vrai ou juste? Si cela est vrai, jusqu'où cette réflexivité s'exerce-t-elle, et est-elle une réflexivité critique ou d'accompagnement d'un ordre des choses qui lui échappe? Plusieurs points d'attention doivent nous alerter.

2-Voir le site alliancevita.org, consulté le 31 mars 2021, qui prône le respect de l'intégrité de l'embryon humain, parce que cet embryon est "l'un des nôtres", le respect de l'enfant alors que l'AMP risque de consacrer un "droit à l'enfant " et "le refus de tout eugénisme, c'est-à-dire de la mise en pratique d'une idéologie qui désignerait des êtres humains inférieurs". Nous soulignons cet accent mis sur la référence au « respect ".

3 - Voir le site du collectif Inter-LGBT et Les Enfants d'Arc-en-ciel, l'asso, consulté le 31 mars 2021, en faveur de l'établissement de la filiation par reconnaissance pour tous les couples de même sexe quelque soit leur statut conjugal, revendiquant d'ouvrir l'accès à l'AMP et à la filiation pour tous et toutes, l'extension du droit commun pour garantir l'égalité de traitement de toutes les familles, l'établissement de la filiation des enfants nés de personnes trans. Sur l'invitation à participer au débat bioéthique en date du 27 février 2018, on peut lire: "Alors que "La Manif pour tous" tente de pourrir le débat en récitant des "arguments" appris par cour, rendons plutôt ce débat concret, vivant, actuel! Mobilisons-nouspour faire entendre notre voix et montrer les difficultés concrètes auxquelles nous sommes confronté.e.s. " 
En effet, le contraste est saisissant entre l'ampleur des questions mises à la discussion et le relatif désintérêt de l'engagement citoyen à leur égard. Si la question fédératrice «Quel monde voulons-nous pour demain? » était placée en exergue du site des États généraux de la bioéthique, la disproportion est énorme entre les enjeux à évaluer et les moyens - osera-t-on dire étiques? - déployés pour les mettre en perspective. Cette disproportion concerne tout d'abord l'effectivité du pilotage d'un système technique coordonné aujourd'hui à l'échelle globale (par exemple, le libre accès aux données pour l'intelligence artificielle, les techniques d'AMP isonormées qui supposent des standardisations techniques internationales) alors qu'il n'y a pas d'instance normative qui aujourd'hui puisse, à cette échelle, véritablement le réguler. Le pouvoir de la norme paraît l'avoir emporté sur celui de la loi. Est-ce provisoire?

Elle porte ensuite sur l'inertie du système technique au sein duquel la biomédecine est installée. Celui-ci, à travers ses schèmes pratiques, pose

\section{La bioéthique}

n'honore pas le

bios de son nom. les enjeux d'une façon qui se trouve aujourd'hui en décalage à l'égard de la conscience éthique que nous en avons. Ainsi la bioéthique n'honore pas le bios de son nom. Elle est essentiellement éthique médicale, mais ne pense pas intimement les relations entre éthiques médicale, animale et environnementale qu'impose la situation écologique - sans parler de l'actuelle pandémie. Là aussi, une téléologie implicite fait de l'innovation technique une nouvelle figure du destin - «On n'arrête pas le progrès ». Au risque de la confusion entre la catégorie descriptive d'innovation et la catégorie évaluative de progrès.

L'uniformisation des standards de production à l'échelle internationale prépare en outre une unidimensionnalisation des manières éthiques de le réguler. Cela tient à ce que robotique, intelligence artificielle ou biotechnologies de séquençage de génome à haut débit sont des innovations techniques soutenues et portées par des stratégies industrielles et des options (au sens de stock-options) financières colossales qui, au-delà même de leur puissance d'influence, sont loin d'être neutres éthiquement. Il y a bien là une fin des moyens, qui valorisent la vitesse, la fiabilité, la transparence, l'efficience, l'individualité (dans l'idée de médecine dite personnalisée mais non nécessairement personnalisante). Et proposent, ce faisant, une certaine conception de ce que seraient une vie bonne et une société juste. Cette dernière entre en discussion avec d'autres manières 
de concevoir l'éthique, qui, elles, n'ont pas les « moyens » de ces stratégies économiques et industrielles. Celles-ci soutiennent l'importance de la lenteur de la réflexion, de la confiance, de la complexité, de la gratuité et de l'individuation personnelle non confondue avec l'individualisation. Que peut la loi bioéthique si, prise dans ce grand mouvement d'innovation, elle l'accompagne plutôt que de l'évaluer de l'extérieur en en contestant les normes? Devant l'ampleur de ce qu'engagent des stratégies industrielles et économiques, à quelles conditions l'éthique peut-elle vraiment jouer son rôle d'évaluation critique sans être instrumentalisée pour ne devenir qu'un accompagnement social? Cela n'exige-t-il pas qu'elle soit installée en amont et au cœur des projets et non seulement en aval? On pense ici au titre VI du projet de loi parlant d'une gouvernance éthique " adaptée au rythme des avancées rapides des sciences et des techniques ${ }^{4}$ 》...

Enfin, nous vivons encore en ces matières une dichotomie entre démocratie représentative et démocratie participative. En témoigne l'organisation de débats laissés à l'initiative des espaces de réflexion éthique régionaux, au sein desquels les parlementaires participants avaient du mal à ne pas tenir une position d'autorité. De même, la consultation en ligne n'a guère corrigé les défauts reconnus à la question des liens entre représentation nationale et représentativité. À titre d'exemple, sur le site des États généraux, il y eut 3527 contributions, 49275 votes et 4090 participants sur le thème des examens génétiques et de la médecine génomique; 2034 contributions, 21559 votes, 2130 participants sur «Santé et environnement »; 1853 contributions, 21355 votes, 2339 participants sur la robotique soignante et l'intelligence artificielle, et enfin sur « Procréation et société », de loin le débat le plus suivi, 29128 contributions, 317406 votes, 12338 participants. Ces petits nombres laissent sceptiques quant aux liens entre démocratie sanitaire, démocratie participative et représentative. L'idéal de démocratie directe que réaliserait la consultation en ligne montre les limites de cette dernière: le lobbying n'assure pas la représentativité des votants et nuit à la transparence; la capacité à participer est inégale; les internautes participants sont souvent

4-Le Gouvernement a déposé un projet de loi le 24 juillet 2019 qui comprend sept titres dont le Titre VI (articles 29 et 30) : assurer une gouvernance bioéthique adaptée au rythme des avancées rapides des sciences et des techniques. 
les plus favorisés ou déjà concernés; l'intérêt pour certains thèmes est privilégié aux dépens d'autres qui s'en sont trouvés invisibilisés ${ }^{5}$.

\section{Une mise en travail de nos puissances d'agir}

Le réexamen de ces lois bioéthiques interroge nos capacités d'initiative et d'action. En matière d'initiative, il révèle d'abord une impuissance, liée au désintérêt à l'égard du déploiement de nouvelles techniques qui vont pourtant avoir un impact considérable sur la manière d'envisager le soin - robotique, IA, génomique. Il questionne les raisons de vouloir maintenir une relation humaine de pleine présence au centre des interactions soignant-soigné, avec l'essor du numérique. Tout semble se passer comme si ces enjeux étaient jugés trop impersonnels et démobilisaient en raison de la nécessité de leur déploiement, sur laquelle nous n'aurions aucune prise. En matière d'action, a contrario, il y a davantage un surinvestissement - il ne s'agit pas, disant cela, de ne pas reconnaître les drames ou les situations de vie concrètes qu'elles concernent - de deux questions très spécifiques : la procréation et la fin de vie. Elles ont focalisé l'attention, monopolisé les débats et les énergies ${ }^{6}$, laissant se développer une relative placidité quant aux autres enjeux. Affirmant que le corps est politique, dans la double dimension du sexe, via la procréation, et de la mort, via la fin de vie, la puissance d'agir dans un monde globalisé retrouve dans l'incarnation des enjeux un levier pour pouvoir réorienter l'avenir, du corps à la fois social et politique. On se trouve là à la jonction entre le sociétal et le social. Non qu'il faille choisir, mais parce qu'il importe de travailler à les articuler si l'on conçoit qu'un monde commun n'est pas qu'une juxtaposition d'intérêts. Le sociétal isole du social ou des enjeux de société, des questions qui littéralement prennent corps dans de vitales situations d'existence, qu'elles concernent l'engendrement, l'identité personnelle, la mort. Il incarne les enjeux, attire l'attention sur la singularité de formes de vies humaines qui demandent à être reconnues

5 -Voir Sophie Monnier, «La consultation en ligne dans le cadre des États généraux de la bioéthique: un progrès démocratique en demi-teinte ", communication au colloque " Le pétitionnement électronique ", Lille, 27 mars 2019.

6 - Le site internet des États généraux de la bioéthique décompte près de 183500 visiteurs uniques; 29032 participants ont posté 64985 contributions. Les thématiques "Procréation et société » et "Prise en charge de la fin de vie » ont réuni $69 \%$ du total des contributions. 
contre l'abstraction des principes, d'où la véhémence et la puissance de la volonté qui animent les acteurs de ces questions sociétales. Mais le sociétal n'est pas sans le social, qui déploie un cadre où vivre, instituer ces demandes. Les questions les plus intimes sur ce qui fait le désir d'enfant, l'identité sexuelle, une vie jusqu'à la mort, une hybridation homme/ machine sont des questions éminemment sociales et politiques, les institutions leur offrant le cadre où se déployer. La bioéthique est, eu égard à la brutalité de l'opposition pro life et à la revendication de reconnaissance qu'elle tente d'arbitrer au sein d'un État «métaphysiquement neutre » dirait Habermas, l'un des opérateurs de traduction du sociétal en social.

En termes de puissances d'agir, ce réexamen est le rendez-vous manqué des relations entre santé et environnement, alors que, pendant cette séquence législative, se vote une « loi climat » et se déploie une pandémie qui rappelle dramatiquement l'importance des relations entre médecine et écologie. Le corps est politique et la Covid-19 a donné une ampleur mondiale à cette affirmation. Nous pensons encore les relations entre santé et environnement comme des relations d'extériorité et pas assez d'interrelations, lesquelles invitent à repenser notre conception du corps. Le dualisme âme/corps qui soutient l'épistémologie biomédicale - notre corps est comme une machine horloge ou ordinateur - repose sur un dualisme nature/culture. Il maintient l'idée que la nature n'est qu'un décor et non un milieu, juxtaposant des enjeux qu'il faudrait penser ensemble. Outre la protection effective des écosystèmes dont la dégradation pèse lourdement sur la santé via les zoonoses, les infertilités, la multiplication des anomalies génétiques et des cancers, il s'agirait d'explorer en profondeur les liens entre écologie et bioéthique. Le défi anthropologique est de proposer de la bioéthique non une définition faible centrée sur l'humain seul, mais une définition forte: ce que signifie pour l'humain être vivant parmi les vivants. C'est la compréhension de notre corps qui est engagée là, dans les relations entre santé et environnement. Si en matière d'écologie, la conscience de notre appartenance corporelle au monde semble faire consensus (pandémie oblige!), en matière d'éthique médicale, le statut du corps fait l'objet d'oppositions franches: naturalisation du corps, constructivisme social, mobilisation équivoque de l'«écologie intégrale ». Que signifie par exemple «intégral»? Est-ce l'intégrisme d'une nature immuable définissant la norme, ou l'intégrité d'une conception relationnelle de l'autonomie humaine? 
Un dernier lieu de déploiement des puissances d'agir concerne la place des religions dans le débat bioéthique animé par et dans une société de sécularisation avancée. Le réexamen des lois a vu les traditions religieuses, et plus particulièrement la frange des catholiques du mouvement La Manif pour tous, s'opposer extrêmement violemment et d'une façon très peu évangélique à ces projets de loi. Elle exalte la Vie aux dépens des vies, la Nature aux mépris des histoires, la Vérité morale définitive dans l'indifférence au discernement éthique de situations difficiles. Ces prises de position très peu «catholiques », soutenues par des discours identitaires, masquent que nombre de croyants, dont des catholiques, ont été des acteurs de ces débats bioéthiques, occupant des positions de médiation et non de clivage. Beaucoup ont intégré, dans une pudeur läque, une manière d'exprimer des convictions sur un mode critique. La bioéthique opère alors comme un révélateur entre, d'un côté, une conception de la religion qui rêve encore de pouvoir imposer une vision du monde à partir de principes moraux intransigeants et anhistoriques, et, d'un autre côté, une manière de contribuer à enrichir la réflexion éthique, en mobilisant les ressources sémantiques et symboliques des traditions religieuses, non pour maintenir l'ordre mais pour imaginer autrement la joie d'être. Ces traditions sont convoquées comme éléments contribuant à éclairer le débat public et non pour le clore.

On ne peut faire porter aux seules lois bioéthiques le lourd fardeau d'avoir à s'orienter avec justice et justesse dans des sociétés mobiles, complexes et différenciées comme le sont les nôtres. Mais on doit discuter toutefois le retard dans la mise en œuvre des lois antérieurement votées (vingt départements français n'ont pas de soins palliatifs et la plupart des Français favorables à l'« euthanasie » ignorent qu'ils peuvent rédiger des directives anticipées et désigner une personne de confiance). Entre la finalité longue d'une explicitation du type de monde que nous désirons et celle du temps court que représente ce moment d'écriture des lois de bioéthique, il est, entre les deux, des institutions, des associations, des organisations, des entreprises, des partis qui sont autant d'opérateurs de traduction de la première dans la seconde, et réciproquement. À côté de la bioéthique, il y a d'autres conseils ou comités éthiques: du CESE à la CNIL et autres structures syndicales, organisations patronales, etc. C'est à ces endroits qu'il conviendrait de lire les effets de la loi bioéthique et sa traduction en pratiques, arts et manières. 
Les lois bioéthiques n'ignorent pas combien la santé est devenue une industrie. Elles ont une fonction d'alerte sur les points d'attention de ce qui de l'homme et de l'autre homme ne saurait être discuté ou transigé. Ultimement, n'est-ce pas cette fonction de protestation et d'attestation que ces lois bioéthiques font exister, au sein du social, à partir de ce tiers lieu imaginatif qu'est la réflexion éthique? 
